\title{
Evaluation of excessive transmission line losses caused by unbalanced and nonlinear three-phase loads
}

\author{
${ }^{1}$ Klemen Deželak, ${ }^{1}$ Gorazd Štumberger \\ ${ }^{1}$ University of Maribor, Faculty of Electrical Engineering and Computer Science \\ Smetanova 17, 2000 Maribor, Slovenia \\ phone:+386 2220 7180, fax:+386 2220 7272, e-mail: klemen.dezelak.@uni-mb.si
}

\begin{abstract}
This work evaluates excessive transmission line losses caused by unbalanced and nonlinear three-phase loads. The orthogonal decomposition of currents is applied in order to determine current component indispensable for energy transmission and current components that do not contribute to the energy transmission. The last ones cause excessive losses in the transmission lines which can be avoided.
\end{abstract}

Key words: transmission line losses, orthogonal current decomposition, asymmetrical and nonlinear three-phase loads

\section{Introduction}

This work evaluates excessive transmission line losses in the three-phase systems caused by unbalanced and nonlinear loads. In the single-phase systems, where currents and voltages contain exclusively fundamental harmonic components, energy transmission and reciprocal energy exchange between source and load can be adequately treated by the concept of active and reactive power [1]. However, this approach fails when currents and voltages contain higher order harmonic components or when unbalanced and nonlinear threephase loads are analyzed. In such cases mathematical tools like orthogonal decompositions of currents in the time or frequency domains [2]- [8] can be applied. Using these tools, it is possible to determine current components indispensable for energy transmission and those current components that do not contribute to the energy transmission and cause excessive transmission losses which can be avoided.

In this work, the orthogonal decomposition of threephase currents in the time domain is used to determine current component indispensable for energy transmission. The ratio between its RMS (root mean square) value and the RMS value of the original three-phase current is applied to introduce generalized power factor in the three-phase system. The generalized power factor is used to characterize the load unbalance and effects of nonlinear load. Excessive transmission losses in a low voltage distribution line are evaluated by case studies performed for different operating conditions.

\section{Orthogonal decomposition of currents}

In this section current and voltage vectors of the threephase system are introduced together with the inner product. They are used to define the current vector component indispensable for three-phase energy transmission and the generalized power factor of the three-phase system.

Let $i_{1}(t), i_{2}(t), i_{3}(t)$ and $u_{1}(t), u_{2}(t), u_{3}(t)$ be the line currents and voltages of a three-phase system observed inside a selected time window $[0, T]$. Let us introduce the current vector $\mathbf{i}(\mathrm{t})$ and the voltage vector $\mathbf{u}(\mathrm{t})$ by (1).

$$
\mathbf{i}(t)=\left[\begin{array}{l}
i_{1}(t) \\
i_{2}(t) \\
i_{3}(t)
\end{array}\right], \mathbf{u}(t)=\left[\begin{array}{l}
u_{1}(t) \\
u_{2}(t) \\
u_{3}(t)
\end{array}\right]
$$

The inner product of current and voltage vectors is defined by (2):

$$
\begin{aligned}
(\mathbf{u}, \mathbf{i}) & =\frac{1}{t_{2}-t_{1}} \int_{t_{1}}^{t_{2}} \mathbf{u}^{T} \mathbf{i} d \tau=\frac{1}{t_{2}-t_{1}} \int_{t_{1}}^{t_{2}}\left[\begin{array}{l}
u_{1}(\tau) \\
u_{2}(\tau) \\
u_{3}(\tau)
\end{array}\right]^{T}\left[\begin{array}{l}
i_{1}(\tau) \\
i_{2}(\tau) \\
i_{3}(\tau)
\end{array}\right] d \tau= \\
& =\frac{1}{t_{2}-t_{1}} \int_{t_{1}}^{t_{2}} p_{1}(\tau)+p_{2}(\tau)+p_{3}(\tau) d \tau=P_{1}+P_{2}+P_{3}=P
\end{aligned}
$$

where $p_{1}, p_{2}$ and $p_{3}$ denote instantaneous values of power in all three phases. From (2) it is obvious that the inner product of current and voltage vectors equals the average active power $P$ of the three-phase system which is given as a sum of average active powers in individual phases denoted by $P_{1}, P_{2}$ and $P_{3}$.

Let us introduce RMS values of the current vector $I$ and voltage vector $U$ by Euclidean norms of these vectors (3) and (4). Squares of both norms equal the sums of squared current or voltage RMS values in individual phases. 


$$
\begin{gathered}
I^{2}=\|\mathbf{i}\|^{2}=I_{1}^{2}+I_{2}^{2}+I_{3}^{2}=\frac{1}{t_{2}-t_{1}} \int_{t_{1}}^{t_{2}} \mathbf{i}^{T} \mathbf{i} d \tau= \\
=\frac{1}{t_{2}-t_{1}} \int_{t_{1}}^{t_{2}} i_{1}(\tau) i_{1}(\tau)+i_{2}(\tau) i_{2}(\tau)+i_{3}(\tau) i_{3}(\tau) d \tau \\
U^{2}=\|\mathbf{u}\|^{2}=U_{1}^{2}+U_{2}^{2}+U_{3}^{2}=\frac{1}{t_{2}-t_{1}} \int_{t_{1}}^{t_{2}} \mathbf{u}^{T} \mathbf{u} d \tau= \\
=\frac{1}{t_{2}-t_{1}} \int_{t_{1}}^{t_{2}} u_{1}(\tau) u_{1}(\tau)+u_{2}(\tau) u_{2}(\tau)+u_{3}(\tau) u_{3}(\tau) d \tau
\end{gathered}
$$

In (3) and (4) $I_{1}, I_{2}, I_{3}$, and $U_{1}, U_{2}, U_{3}$ denote the RMS values of currents and voltages in all three phases. The equivalent conductivity of entire three-phase system $G$ is defined by (5).

$$
G=\frac{P}{U^{2}}=\frac{P}{\|\mathbf{u}\|^{2}}
$$

The equivalent conductivity $G(5)$ is required to introduce current vector $\mathbf{i}_{u}$ (6) which is collinear with applied voltage vector $\mathbf{u}$ and is the only current vector indispensable for energy transmission.

$$
\mathbf{i}_{u}=G \mathbf{u}=\frac{P}{U^{2}} \mathbf{u}
$$

The definition of current vector $\mathbf{i}_{u}$ (6) is general. It is appropriate to be used in three-phase systems with unbalanced and nonlinear load. Considering (1), (2), (3) and (6), the norm (RMS) value of the current vector $\mathbf{i}_{u}$ can be expressed by (7), which leads to (8).

$$
\begin{gathered}
\left\|\mathbf{i}_{u}\right\|^{2}=\left(\mathbf{i}_{u}, \mathbf{i}_{u}\right)=\left(\frac{P}{\|\mathbf{u}\|^{2}} \mathbf{u}, \frac{P}{\|\mathbf{u}\|^{2}} \mathbf{u}\right)=\frac{P^{2}}{\|\mathbf{u}\|^{2}} \\
|P|=\|\mathbf{u}\|\left\|\mathbf{i}_{u}\right\|
\end{gathered}
$$

The generalized power factor of the three-phase system $P F$ ' can be now defined by (9).

$$
P F^{\prime}=\frac{|P|}{\|\mathbf{u}\|\|\mathbf{i}\|}=\frac{\|\mathbf{u}\|\left\|\mathbf{i}_{u}\right\|}{\|\mathbf{u}\|\|\mathbf{i}\|}=\frac{\left\|\mathbf{i}_{u}\right\|}{\|\mathbf{i}\|}
$$

In the first approximation the transmission line losses are proportional to the $\|\mathbf{i}\|^{2}$ while only current vector $\mathbf{i}_{u}$ contributes to the energy transmission. The $\left\|\mathbf{i}_{u}\right\|^{2}$ is proportional to the transmission losses which cannot be avoided. Thus, the square of generalized power factor, actually $1 / P F^{, 2}(10)$, can be used as a measure for excessive transmission losses.

$$
1 / P F^{\prime 2}=\frac{\|\mathbf{i}\|^{2}}{\left\|\mathbf{i}_{u}\right\|^{2}}
$$

\section{Evaluation of excessive transmission losses}

In order to evaluate excessive transmission losses in a low voltage distribution line a system with nonlinear and unbalanced load schematically shown in Figure 1 was applied. The feeder network is denoted by Q while C1 and $\mathrm{C} 2$ denote two measurement points.

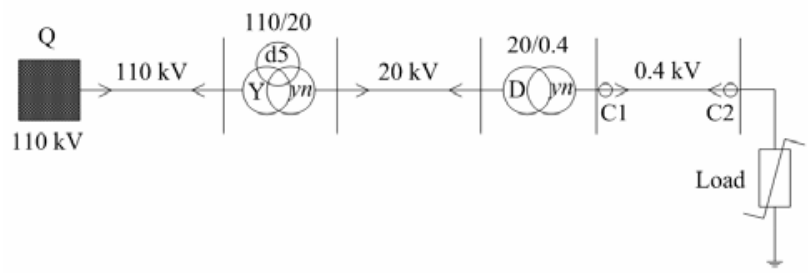

Figure 1: Schematic presentation of discussed system

Transmission losses were evaluated for the following cases:

1. Balanced linear load

2. Unbalanced linear load

3. Balanced nonlinear load

4. Unbalanced nonlinear load

The load power was $72.53 \mathrm{~kW}$ and $131.26 \mathrm{~kW}$ while the generalized power factor was between 0.73 and 1 . The expression "unbalanced" load means that the RMS value of one line current is $20 \%$ higher than the RMS values of the other two line currents.

Figures 2 to 4 show the time dependent line voltages at the beginning $u_{\mathrm{fb}}(\mathrm{C} 1$ in Figure 1$)$ and at the end $u_{\mathrm{fe}}(\mathrm{C} 2$ in Figure 1) of $0.4 \mathrm{kV}$ line together with the corresponding line currents $i_{\mathrm{f}}$.

Figure 2 shows the time behavior of line voltages and currents for a $72.53 \mathrm{~kW}$ linear load. The two upper graphs show currents and voltages for the balanced linear load, while the lower two graphs show the currents and voltages for the unbalanced linear load where the RMS value of one line current is $20 \%$ higher than the RMS values of the other two line currents. The active load power is the same in both presented cases. 

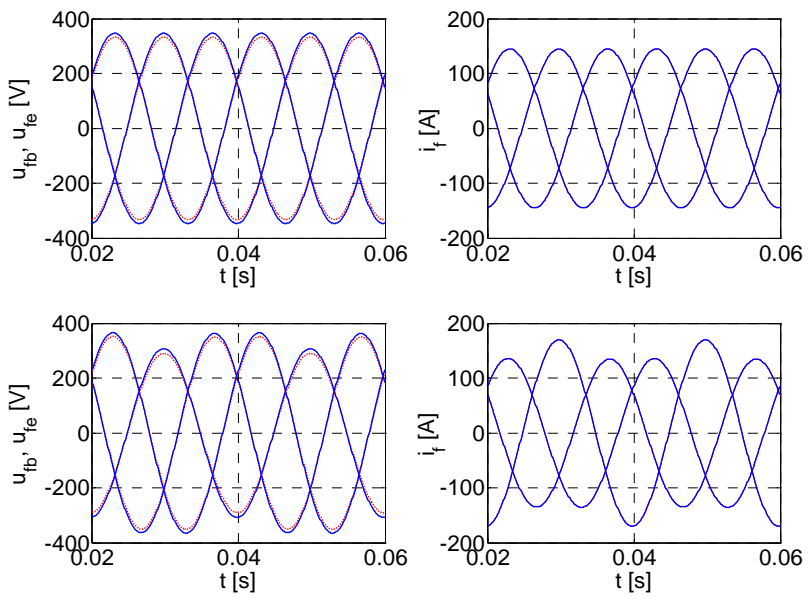

Figure 2: Time behavior of voltage and current given for balanced $72.53 \mathrm{~kW}$ linear load (upper graphs) and for unbalanced $72.53 \mathrm{~kW}$ linear load (lower graphs)

Figure 3 shows the time behavior of line voltages and currents for a nonlinear $72.53 \mathrm{~kW}$ load. The upper graphs are given for the balanced nonlinear load. The lower graphs are given for the unbalanced nonlinear load where the RMS value of one line current is $20 \%$ higher than in the other two lines. The active load power is the same in both presented cases.
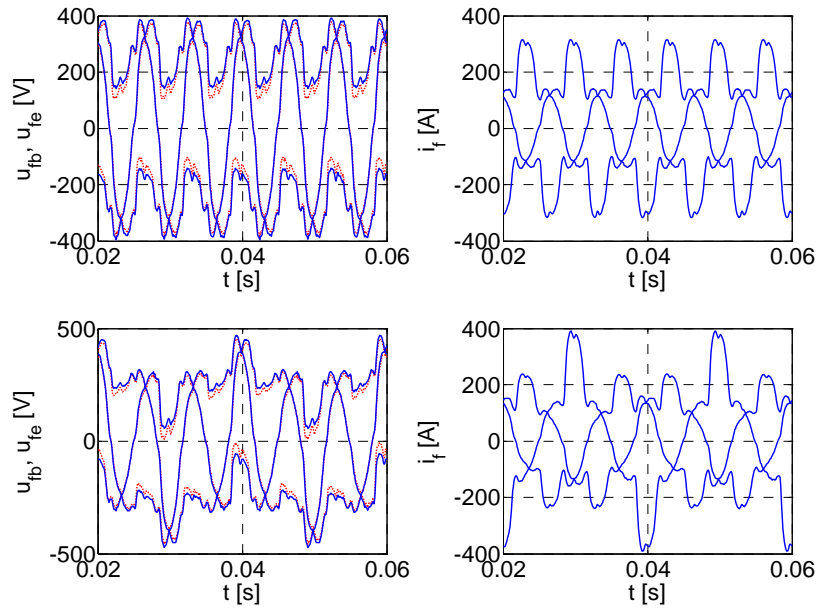

Figure 3: Time behavior of voltage and current given for balanced $72.53 \mathrm{~kW}$ nonlinear load (upper graphs) and for unbalanced $72.53 \mathrm{~kW}$ nonlinear load (lower graphs)

Figure 4 shows the time behavior of line voltages and currents for nonlinear unbalanced load. The upper graphs are given for load with active power $72.53 \mathrm{~kW}$ and generalized power factor 0.94 , while the lower graphs are given for the load with active power $131.26 \mathrm{~kW}$ and generalized power factor 0.8 .
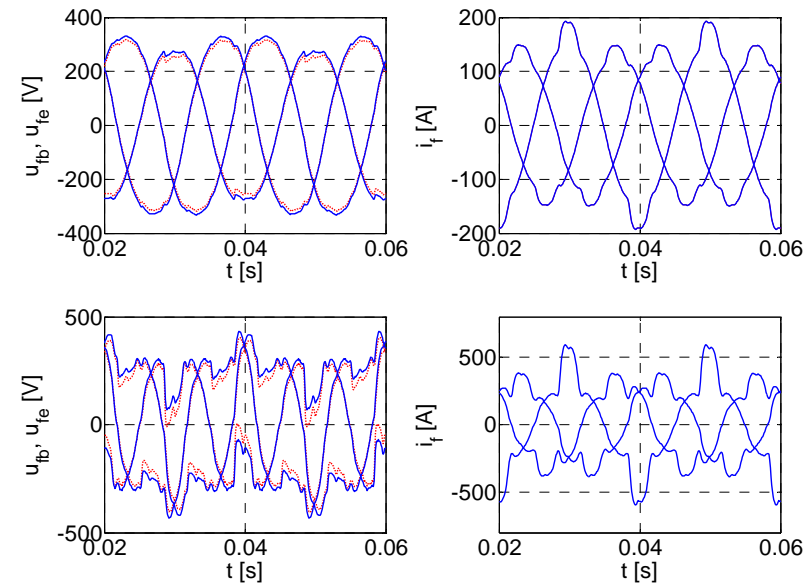

Figure 4: Time behavior of voltage and current given for nonlinear unbalanced load: upper graphs - active power $72.53 \mathrm{~kW}$ and generalized power factor 0.94; lower graphs - active power $131.26 \mathrm{~kW}$ and generalized power factor 0.8 .

Table I shows the relative transmission losses $P_{\text {Loss }}$ calculated as a ratio $\left\|\mathbf{i}\left|{ }^{2} /\right| \mathbf{i}_{u}\right\|^{2}$ (10) for balanced and unbalanced linear loads and balanced and unbalanced nonlinear loads. They are given for different values of the load active power $P_{\text {LOAD }}$ and different values of generalized power factor $P F$ '.

\section{Conclusion}

This paper evaluates excessive transmission line losses caused by unbalanced and nonlinear loads. In order to evaluate these losses in three-phase systems the RMS values of the total current vector current and current vector component indispensable for power transmission must be determined first, which can be done by orthogonal decomposition of currents. The ratio between RMS values of the current vector and the current vector component indispensable for energy transmission can be used to evaluate excessive transmission line losses due to the unbalanced and nonlinear loads. It is shown that the excessive transmission losses increase with the inverse ration of squared generalized power factor. According to the presented results, in the case of unbalanced nonlinear loads the excessive transmission losses can reach almost the same value as the transmission losses due to the linear and balanced loads, which means that the total transmission losses are almost doubled.

\section{References}

[1] S. Svensson, "Power measurement uncertainties in a nonsinusoidal power system,” in International Symposium on Electric Power Engeneering, Proceddings: Power systems, (Stockholm, Sweden), pp. 617-622, IEEE 1995.

[2] L. S. Czarnecki, "Orthogonal Decomposition of the Currents in s 3-Phase Nonlinear Asymmetrical Circuit with a Nonsinusoidal Voltage Source," IEEE Transactions on Instrumentation and Measurement, vol 37, pp 30-34, March 1988.

[3] L. S. Czarnecki, T. Swietlicki, "Powers in nonsinusoidal networks: Their interpretation, analysis, and measurement," IEEE Transactions on Instrumentation and Measurement, vol 39, pp 340-345, April 1990. 
[4] L. S. Czarnecki, "Scattered and reactive current, voltage, and power in circuits with nonsinusoidal waveforms and their compensation," IEEE Trans. on Instr. and Meas., vol. 40, no. 3, pp. 563-567, 1991.

[5] R. Sasdelli, G. C. Montanari, "Compesable power for electrical systems in nonsinusoidal conditions ," IEEE Transactions on Instrumentation and Measurement, vol 43, pp 592-598, April 1994.

[6] L. S. Czarnecki, "Minimisation of unbalanced and reactive currents in three-phase asymmetrical circuits with nonsinusoidal voltage," IEEE Proceedings-B, vol. 139, no. 4, pp. 347-354, 1992.

[7] A. Ferrero, "A new approach to the definition of power components in three-phase systems under nonsinusoidal conditions," IEEE Transactions on Instrumentation and Measurement, vol 40, pp 568-577, Jun 1991.

[8] L. S. Czarnecki, S. M. Hsu, G. Chen "Adaptive balancing compensator," IEEE Transactions on Power Delivery, vol. 10, no. 3, pp. 1244-1250, 1996.

[9] G. Štumberger, B. Polajžer, M. Toman, D. Dolinar, Orthogonal decomposition of currents, power definitions and energy transmission in three-phase systems treated in the time domain, Proceedings of the International conference on renewable energies and power quality (ICREPQ'06), Palma de Mallorca, Spain, April 206.

Table I: Relative transmission losses $P_{\text {Loss }}$ given as a function of active load power $P_{\text {LOAD }}$ and load generalized power factor

PF'.

\begin{tabular}{|c|c|c|c|c|c|c|c|c|}
\hline \multirow{3}{*}{$\begin{array}{c}P_{\mathrm{LOAD}} \\
(\mathrm{kW})\end{array}$} & \multicolumn{4}{|c|}{ Linear load } & \multicolumn{4}{|c|}{ Nonlinear load } \\
\hline & \multicolumn{2}{|c|}{ balanced } & \multicolumn{2}{|c|}{ unbalanced } & \multicolumn{2}{|c|}{ balanced } & \multicolumn{2}{|c|}{ unbalanced } \\
\hline & $P F^{\prime}$ & $P_{\text {LOSS }}(\mathrm{pu})$ & $P F^{\prime}$ & $P_{\text {LOSS }}(\mathrm{pu})$ & $P F^{\prime}$ & $P_{\text {LOSS }}(\mathrm{pu})$ & $P F^{\prime}$ & $P_{\text {LOSS }}(\mathrm{pu})$ \\
\hline 72.53 & 1.000 & 1.000 & 0.979 & 1.042 & 0.754 & 1.757 & 0.728 & 1.888 \\
\hline 72.53 & 1.000 & 1.000 & 0.979 & 1.042 & 0.956 & 1.094 & 0.936 & 1.141 \\
\hline 131.26 & 1.000 & 1.000 & 0.972 & 1.058 & 0.803 & 1.551 & 0.779 & 1.648 \\
\hline
\end{tabular}

